\title{
Insilico Screening of Bioactive Phytochemicals against Spike Protein of COVID-19
}

\author{
Muhammad Hamza Tariq ${ }^{1}$, Mahjabeen Akram $^{2}$ and Yasir Sharif ${ }^{3 *}$ \\ ${ }^{1}$ Atta-ur-Rehman School of Applied Biosciences, National University of Science and \\ Technology, Islamabad, Pakistan \\ ${ }^{2}$ Paediatrics Medicine Unit 1, Services Hospital, Lahore 54000, Pakistan \\ ${ }^{3}$ Institute of Biochemistry and Biotechnology, University of the Punjab, Lahore-54590, \\ Pakistan
}

\begin{abstract}
A B S T R A C T
Coronavirus disease 2019 (COVID-19) is a pandemic and this disease has infected millions of people globally now. COVID-19 is caused by a novel beta coronavirus strain known as severe acute respiratory syndrome coronavirus 2 (SARS-CoV-2). Once SARS-CoV-2 manages to enter the body, it identifies and binds to the angiotensin converting enzyme 2 (ACE2) receptors through the binding receptor of Spike Protein (S-protein). The present study aimed to investigate the phytochemicals as potential inhibitors of the binding domain of S protein so that the binding of COVID-19 with ACE2 receptors could be restrained. For this purpose, the library of 2113 phytochemicals was docked against the binding domain of the S-protein. Top ten compounds with maximum binding affinity to the active sites of target protein were further screened for ADMET properties by adopting SwissADME and ADMETsar online servers. The compounds namely Morin, Curcumin, Apigenin, Cedronolactone A and Matairesinol showed acceptable drug-like properties therefore these compounds can be proposed as effective inhibitors, disrupting the S-protein- ACE2 interaction. This study might help in the development of a natural and cost-effective drug against COVID-19. Further, in vivo and in vitro examinations are required to validate our results.
\end{abstract}

\begin{tabular}{l} 
Article Information \\
\hline Received 10 May 2020 \\
Revised 07 November 2020 \\
Accepted 31 December 2020 \\
Available online 04 November 2021 \\
(early access) \\
Published 17 December 2021 \\
Authors' Contribution \\
\hline YS planned and supervised the \\
research. MHT conducted the research \\
work. MA wrote the article. \\
Key words \\
\hline COVID-19, Phytochemicals, \\
Molecular docking, Spike protein, \\
Druglikeness
\end{tabular}

\section{INTRODUCTION}

S evere acute respiratory syndrome coronavirus 2 (SARSCoV-2) is a novel strain of coronavirus that is reported to cause infectious Coronavirus disease 2019 (COVID-19) (Nadeem et al., 2020). SARS-CoV-2 is characterized by positive stranded non-segmented RNA genome with spike glycoproteins (S-proteins) on its envelope (Walls et al., 2020). These S-proteins gives it crown like appearance under electron microscope. Soon after its incidence it has become a pandemic now, it has affected millions of people globally and the cases are still increasing day by day due to higher degree of virus contagiosity. Pathogenesis of SARS-CoV-2 includes invasion of virus through the respiratory tract by disrupting the intercellular epithelial tight junctions (which are present in human respiratory tract and serve as a first line of defense against foreign invaders). After incursion, SARS-CoV-2 recognizes angiotensin converting enzyme 2 (ACE2) receptors through spike proteins, followed by viral entry into cell, its replication and ultimately severe COVID-19 infection (Zhang et al., 2020). Once binding is accomplished,

\footnotetext{
Corresponding author: s.yashel36@hotmail.com 0030-9923/2022/0001-0433 \$ 9.00/0

Copyright 2022 Zoological Society of Pakistan
}

series of conformational changes start to occur that leads to conversion of S-protein from pre-fusion to a postfusion state. S2 subunit is the other subunit of S-protein that causes the virus to get fused with the cell membrane, allowing it to enter the cell. It is now clear that the entry of SARS-CoV-2 requires ACE2 receptors and these receptors are found in different body cells of all age group human being, however, their level is much more elevated in children, making them more susceptible towards severe form of COVID-19 (Cristiani et al., 2020). Currently there is no therapeutic agent is available to treat this infectious disease while number of infected people is increasing every day.

Active biological agents that are extracted straightforwardly from the plants are called as phytochemicals, these show remarkable sustenance and pharmaceutically effectiveness because of their prominent natural qualities. Phytochemicals have been reported to have very good anti-viral activities with minimum side-effects (Kapoor et al., 2017; Shahzad et al., 2019), therefore, the current study is aimed to identify some bioactive phytochemicals that might be able to act as a potential inhibitor of $\mathrm{S}$ protein so that the attachment of SARS-CoV-2 with ACE2 receptors could be restricted. 


\section{MATERIALS AND METHODS}

\section{Structure retrieval and optimization}

SARS-CoV-2 receptor binding domain was retrieved from Protein databank (Berman et al., 2000) as PDB ID 6W41. This selected protein contains the Crystal structure of SARS-CoV-2 receptor binding domain in complex with human antibody CR3022. This structure was downloaded as PDB file and opened in Molecular Operating Environment (MOE) software. This target protein was first optimized by removing Human receptor and antibody. Human receptor and antibody was removed while receptor binding domain of Spike Protein of SARS-CoV-2 was taken for further study. This target protein was first optimized by; 3D protonation and energy minimization using MOE software and taken as receptor for docking study.

\section{Ligand library preparation}

Three dimensional structure of 2113 bioactive phytochemicals was downloaded from PubChem database (Kim et al., 2015), out of which 227 were alkaloids, 81 were aromatic, 11 were carbohydrates, 999 were flavonoids, 88 were lignans, 63 were polycylclicaromatic, 51 were saponins, 77 were steroids, 6 were tanins and 510 were terpenoids. All these phytochemicals were also optimized by $3 \mathrm{D}$ protonation and energy minimization. A library containing all these optimized phytochemicals was established for docking study.

\section{Molecular docking}

Docking analysis was performed by using Molecular Operating Environment software (Vilar et al., 2008). For this purpose, docking was done between ligand library and active residues of target protein, these active residues were determined by site finder tool of MOE software. Once the docking was completed, phytochemicals with best conformations were identified according to Root Mean Square Deviation (RMSD) value and S-score. RMSD represents the mean distance amongst the backbone atoms of superimposed proteins and S-score is a mathematical value that demonstrates the binding affinity of ligands with their receptors with all potential binding geometries. LigX tool of MOE was adopted to examine the clear vision of $2 \mathrm{D}$ and $3 \mathrm{D}$ plots of receptor ligand interactions.

\section{In silico analysis of drug likeness and ADMET properties}

Top ten Phytochemicals with minimum docking score were further appraised for Lipinski rule of five to evaluate their drug-like attributes by engaging Molinspiration online tool (URL: http://www.molinspiration.com//cgibin/properties). Any compound violating more than two parameters of Lipinski rule was not considered as a good drug against TBX19. All those compounds that followed Lipinski rule of five were further considered for ADMET (Adsorption, Distribution, Metabolism, Excretion and Toxicity) like properties by practicing admetSAR (URL: http://lmmd.ecust.edu.cn/admetsar1) (Cheng et al., 2012) and SwissADME software (URL: http://www.swissadme. ch/) (Daina et al., 2017).

\section{RESULTS AND DISCUSSION}

Drug designing has been radically revolutionized due to in silico analysis and bioinformatics. It has decreased both cost and time required for drug discovery. Due to advancements in Chemoinformatics, libraries of 3D structures of compounds are accessible that could be tested for their potential therapeutic effects via different computational tools. Molecular Docking involves the Insilco estimation of best suitable three-dimensional conformation of ligand- target complex and also predict the free energy of resulting complex (Bortolato et al., 2013), therefore, it is considered as a common protocol in drug-design (de Ruyck et al., 2016). The current study employed a library of 2113 bioactive phytochemicals to evaluate their ability to inhibit the receptor binding domain of S-protein. For this purpose, docking analysis was performed between phytochemical library and receptor binding domain of S-protein.

\section{Docking analysis}

Library of energy minimized 2113 phytochemicals was docked with receptor binding domain of COVID-19 spike protein. Once the docking was completed, phytochemicals were ranked on the basis of four different parameters i.e. minimum Gibbs free energy, maximum occupancy of the binding pocket and Power of different binding forces. Out of 2113 phytochemicals, top ten were selected on the basis of lower RMSD value, minimum S-score and maximum binding site occupancy (Table I). All of these ten compounds had an S-score within the range of $-14.49 \mathrm{kcal} / \mathrm{mol}$ to $-11.64 \mathrm{kcal} / \mathrm{mol}$. These ten compounds include; Tannic acid, Oenin, Morin, Chebulinic Acid, Uncarinic Acid C, Cedronolactone A, Matairesinol, Apigenin, Curcumin, D-Chicoric acid. Amino acids; Phe 342, Ala 344, Asp 363 and Ser 373 were found to be most active amino acid residues as they showed interactions with three out of ten phytochemicals.

\section{Evaluation of drug-like properties}

These ten compounds were further assessed for druglike properties by considering them for Lipinski rule of five according to which a compound shows drug like properties must have a molecular weight of $<500 \mathrm{Da}$, it should 
contain $<5$ Hydrogen bond Donor (HBD), <10 Hydrogen Bond Acceptor (HBA) and lipophilicity of $\mathrm{ClogP}<05$ (Lipinski et al., 1997). Out of ten, only five compounds followed this rule namely; Morin, Matairesinol, Apigenin, Cedronolactone A and Curcumin (Table II) with binding energy of $-12.6072,-12.0830,-11.9076,-11.8840$ and -11.8678 respectively. Curcumin is a flavonoid that is derived from Curcuma longa plant. It has been reported as a therapeutic substance against different viruses including; Chikungunya, dengue and Zika viruses (Mounce et al., 2017). Apigenin is also a flavonoid, produced by Ocimum basilicum. It is also known for its anti-viral activity against african swine fever virus, hepatitis $B$ virus and adenoviruses (Chiang et al., 2005). Morin is also a flavonoid that is isolated from Maclura pomifera and reported for its activity against Equid herpesvirus 1 (Gravina et al., 2011). Matairesinol is a plant lignin whereas Cedronolactone A is terpenoid in nature, both of have no reported anti-viral activity however their bioactivity is well known (Shoeb et al., 2004; Guo et al., 2005).

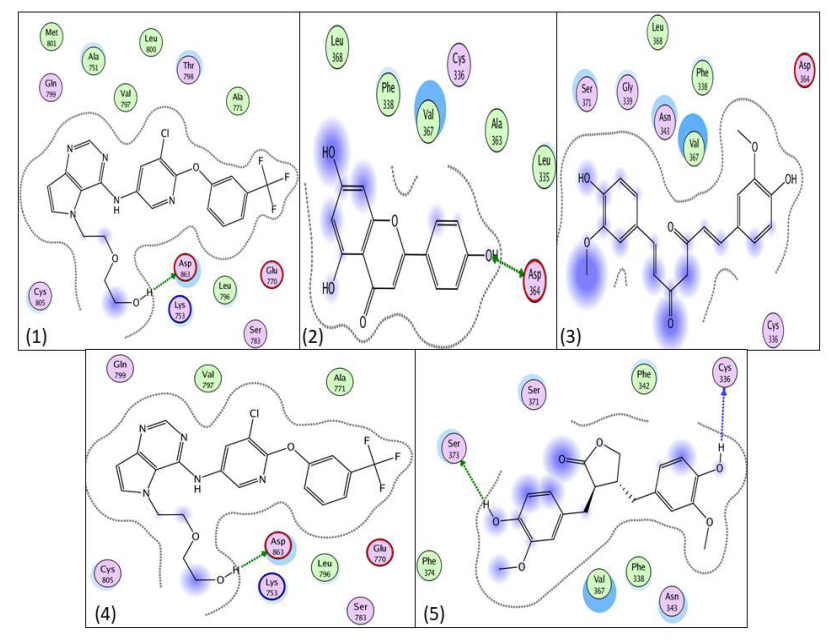

Fig. 1. 2D interaction images of top five phytochemicals, having potential drug-like properties, against COVID-19 spike protein where (1) shows interactions of Morin in which side chain atom, Asp 863 is involved in hydrogen bond interaction, (2) shows interactions of Apigenin in which side chain atom Asp 364 is involved in hydrogen bond formation, (3) shows interactions of Curcumin in which Asp 364 is involved in hydrogen bond formation, (4) shows interactions of Cedronolactone A in which Asp 863 is involved in hydrogen bond interaction and (5) shows interactions of Matairesinol in which Ser 373 and Cys 336 are involved in hydrogen bond interaction.

These five compounds were then assessed for ADMET attributes so that their potential drug-like properties could be evaluated, results of which are shown in Table II.
Among ADMET properties; Blood Brain Barrier (BBB) represents the capability of a drug to cross BBB, Gastrointestinal (GI) tract displays that how well the drug is absorbed in GI tract whilst Caco-2 permeability depicts the ability of a drug to get absorbed in intestine. All of the five compounds were non-carcinogenic in nature and non-permeant towards BBB. All of them were found to be highly absorbent in GI tract. Apigenin, Matairesinol and Cedronolactone A were Caco-2 Permeable whereas Morin and Curcumin were non-permeable for Caco-2.

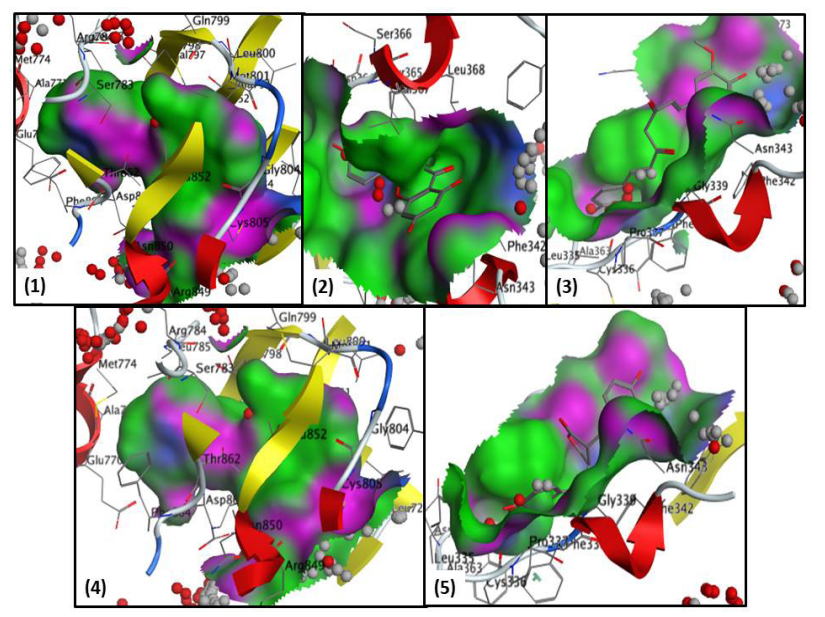

Fig. 2. 3D interaction images of top five phytochemicals, having potential drug-like properties, against COVID-19 spike protein where (1) shows interactions of Morin (2) shows interactions of Apigenin (3) shows interactions of Curcumin (4) shows interactions of Cedronolactone A and (5) shows interactions of Matairesinol.

Molecular Docking analysis has been used previously to predict potential therapeutic agent of COVID-19. Hall and Ji (2020) performed the docking analysis between library of different compounds and two target proteins of COVID-19 i.e. spike glycoprotein, and the 3CL protease. Thuy et al. (2020) also performed docking between phytochemicals of garlic (Allium sativum) essential oil and ACE2 receptors of human and main protease enzyme of COVID-19.

\section{CONCLUSION}

The COVID-19 is an infectious disease that has infected millions of people globally and it is still spreading all over the world. There is no well-known therapeutic agent currently available to treat this disease. The current study proposed that morin, curcumin, apigenin, cedronolactone A and matairesinol are the phytochemicals that have a potential to constrain the attachment of SARS- 
Table I. Top ten phytochemicals with minimum S-score and maximum binding capabilities for spike protein of COVID-19, generated with MOE software.

\begin{tabular}{llllll}
\hline Sr. no & Pubchem ID & Phytochemical name & S-score & Rmsd value & Interacting residues \\
\hline 1 & 16129778 & Tannic Acid & -14.4967 & 3.9527 & Ala 344, Phe 342, Arg 509, Cys 336 \\
2 & 72284 & Chebulinic Acid & -13.9819 & 2.2131 & Ala 344, Asn 343, Ser 371 \\
3 & 443652 & Oenin & -13.2615 & 2.0824 & Ser 373, Asn 343, Phe 342 \\
4 & 44583694 & Uncarinic Acid C & -12.9015 & 1.8111 & Asp 863 \\
5 & 5281670 & Morin & -12.6072 & 1.3346 & Asp 863 \\
6 & 119205 & Matairesinol & -12.0830 & 1.3719 & Ser 373 \\
7 & 5280443 & Apigenin & -11.9076 & 0.7896 & Asp 364 \\
8 & 11754814 & Cedronolactone A & -11.8840 & 1.3047 & Asp 863 \\
9 & 969516 & Curcumin & -11.8678 & 0.5795 & Asp 364 \\
10 & 5470299 & D-Chicoric Acid & -11.6446 & 2.4099 & Ala 344, Phe 342, Arg 509, Ser 373 \\
\hline
\end{tabular}

Table II. Results of top 10 phytochemicals, examined for Lipinski rule of five by using Molinspiration.

\begin{tabular}{lllll}
\hline Phytochemical & $\begin{array}{l}\text { Molecular weight (g/ } \\
\text { mol) }\end{array}$ & $\begin{array}{l}\text { Number of hydrogen bond } \\
\text { acceptor }\end{array}$ & $\begin{array}{l}\text { Number of hydrogen bond } \\
\text { donor }\end{array}$ & MLogP \\
\hline Lipinski rule of five & $<500$ & $<10$ & $<05$ & $<05$ \\
Tannic acid & 1701.21 & 46 & 25 & 7.06 \\
Chebulinic acid & 956.11 & 27 & 13 & -0.36 \\
Oenin & 493.44 & 12 & 7 & 0.48 \\
Uncarinic acid C & 632.88 & 6 & 3 & 7.57 \\
Morin & 302.04 & 7 & 5 & 1.64 \\
Matairesinol & 358.14 & 6 & 2 & 2.03 \\
Apigenin & 270.05 & 5 & 3 & 3.22 \\
Cedronolactone A & 478.22 & 9 & 3 & 0.48 \\
Curcumin & 368.13 & 6 & 2 & 2.83 \\
D-Chicoric acid & 474.08 & 12 & 6 & 1.12 \\
\hline
\end{tabular}

Table III. ADMET profiling enlisting absorption, metabolism and toxicity related drug like parameters of candidate compounds.

\begin{tabular}{llllll}
\hline Phytochemical & Morin & Apigenin & Curcumin & Matairesinol & Cedronolactone A \\
\hline A. Absorption & & & & & No \\
Blood-brain barrier permeant & No & No & No & No & High \\
Gastro- intestinal absorption & High & High & High & High & No \\
Caco-2 permeability & No & Yes & No & Yes & Yes \\
P-glycoprotein substrate & No & No & No & No & No \\
B. Metabolism & & & & No & No \\
CYP450 1A2 inhibitor & Yes & Yes & No & No & No \\
CYP450 2C19 inhibitor & No & No & No & No & No \\
CYP450 2C9 inhibitor & No & No & Yes & Yes & Yes \\
CYP450 2D6 inhibitor & Yes & Yes & No & & No \\
CYP450 3A4 inhibitor & Yes & Yes & Yes & Yes & No \\
C. Toxicity & & & & No & No \\
Human either-a-go-go inhibition & No & No & No & Yes & No \\
Carcinogens & No & No & No & No & \\
AMES mutagenesis & Yes & & &
\end{tabular}


CoV-2 with human body cells, thereby preventing the body from being infected by it. Additional in vitro and in vivo experiments are highly advised to assess the efficacy of above mentioned phytochemicals.

\section{Statement of conflict of interest}

The authors have declared no conflict of interest.

\section{REFERENCES}

Berman, H.M., Westbrook, J., Feng, Z., Gilliland, G., Bhat, T.N., Weissig, H., Shindyalov, I.N. and Bourne, P.E., 2000. The protein data bank. Nucl. Acids Res., 28: 235-242. https://doi.org/10.1093/ nar/28.1.235

Bortolato, A., Fanton, M., Mason, J.S. and Moro, S., 2013. Molecular docking methodologies. Meth. mol. Biol., 924: 339-360. https://doi.org/10.1007/978-162703-017-5_13

Cheng, F., Li, W., Zhou, Y., Shen, J., Wu, Z., Liu, G., Lee, P.W. and Tang, Y., 2012. Admetsar: A comprehensive source and free tool for assessment of chemical admet properties. ACS Publications. https://doi.org/10.1021/ci300367a

Chiang, L.C., Ng, L.T., Cheng, P.W., Chiang, W. and Lin, C.C., 2005. Antiviral activities of extracts and selected pure constituents of ocimum basilicum. Clin. exp. Pharmacol. Physiol., 32: 811-816. https://doi.org/10.1111/j.1440-1681.2005.04270.x

Cristiani, L., Mancino, E., Matera, L., Nenna, R., Pierangeli, A., Scagnolari, C. and Midulla, F., 2020. Will children reveal their secret? The coronavirus dilemma. Eur. Respir. J., 55: 2000749. https://doi. org/10.1183/13993003.00749-2020

Daina, A., Michielin, O. and Zoete, V., 2017. Swissadme: A free web tool to evaluate pharmacokinetics, drug-likeness and medicinal chemistry friendliness of small molecules. Sci. Rep., 7: 42717. https://doi. org/10.1038/srep42717

de Ruyck, J., Brysbaert, G., Blossey, R. and Lensink, M.F., 2016. Molecular docking as a popular tool in drug design, an in silico travel. Adv. appl. Bioinform. Chem., 9: 1. https://doi.org/10.2147/ AABC.S105289

Gravina, H., Tafuri, N., Júnior, A.S., Fietto, J., Oliveira, T., Diaz, M. and Almeida, M., 2011. In vitro assessment of the antiviral potential of transcinnamic acid, quercetin and morin against equid herpesvirus 1. Res. Vet. Sci., 91: 158-162. https:// doi.org/10.1016/j.rvsc.2010.11.010

Guo, Z., Vangapandu, S., Sindelar, R., Walker, L. and Sindelar, R., 2005. Biologically active quassinoids and their chemistry: Potential leads for drug design. Curr. med. Chem., 12: 173-190. https://doi. org/10.2174/0929867053363351

Hall Jr, D.C. and Ji, H.-F., 2020. A search for medications to treat covid-19 via in silico molecular docking models of the sars-cov-2 spike glycoprotein and 3cl protease. Travel Med. Infect. Dis. 35: 101646. https://doi.org/10.1016/j.tmaid.2020.101646

Kapoor, R., Sharma, B. and Kanwar, S., 2017. Antiviral phytochemicals: An overview. Biochem. Physiol., 6: 7. https://doi.org/10.4172/2168-9652.1000220

Kim, S., Thiessen, P.A., Bolton, E.E., Chen, J., Fu, G., Gindulyte, A., Han, L., He, J., He, S. and Shoemaker, B.A., 2015. Pubchem substance and compound databases. Nucleic Acids Res., 44: D1202-D1213. https://doi.org/10.1093/nar/gkv951

Lipinski, C.A., Lombardo, F., Dominy, B.W. and Feeney, P.J., 1997. Experimental and computational approaches to estimate solubility and permeability in drug discovery and development settings. $A d v$. Drug Deliv. Rev., 23: 3-25. https://doi.org/10.1016/ S0169-409X(96)00423-1

Mounce, B.C., Cesaro, T., Carrau, L., Vallet, T. and Vignuzzi, M., 2017. Curcumin inhibits zika and chikungunya virus infection by inhibiting cell binding. Antivir. Res., 142: 148-157. https://doi. org/10.1016/j.antiviral.2017.03.014

Nadeem, M., Shah T.A., Waseem, M., Wani, J.I., Sabah, Z.U. and Javeed, S., 2020. Sars-cov-2, pandemic covid 19: A brief review. Ann. Med. Hlth. Sci. Res., 10: $846-856$.

Shahzad, M.I., Ashraf, H., Arshad, M., Parveen, S., Aslam, A., Naz, N., Kamran, Z., Khalid, S.G., Hameed, S. and Ashfaq, M., 2019. Study of antiviral potential of cholistani plants against new castle disease virus. Pakistan J. Zool., 51. https:// doi.org/10.17582/journal.pjz/2019.51.1.SC11

Shoeb, M., Rahman, M.M., Nahar, L., Delazar, A., Jaspars, M. and Macmanus, S.M., 2004. Bioactive lignans from the seeds of centaurea macrocephala. DARU J. Pharm. Sci., 12: 87-93.

Thuy, B.T.P., My, T.T.A., Hai, N.T.T., Hieu, L.T., Hoa, T.T., Thi Phuong Loan, H., Triet, N.T., Anh, T.T.V., Quy, P.T. and Tat, P.V., 2020. Investigation into sars-cov-2 resistance of compounds in garlic essential oil. ACS Omega, 5: 8312-8320. https:// doi.org/10.1021/acsomega.0c00772

Vilar, S., Cozza, G. and Moro, S., 2008. Medicinal chemistry and the molecular operating environment (moe): Application of qsar and molecular docking to drug discovery. Curr. top. med. Chem., 8: 1555-1572. https://doi.org/10.2174/156802608786786624 
Walls,A.C.,Park, Y.J., Tortorici, M.A., Wall,A., McGuire, A.T. and Veesler, D., 2020. Structure, function, and antigenicity of the sars-cov-2 spike glycoprotein. Cell, https://doi.org/10.1101/2020.02.19.956581

Zhang, H., Penninger, J.M., Li, Y., Zhong, N. and
Slutsky, A.S., 2020. Angiotensin-converting enzyme 2 (ace2) as a sars-cov-2 receptor: Molecular mechanisms and potential therapeutic target. Intens. Care Med., 46: 586-590. https://doi. org/10.1007/s00134-020-05985-9 